\title{
Surge and Choke Capable Compressor Model
}

\author{
Oskar Leufven and Lars Eriksson* \\ * Vehicular Systems, Department of Electrical Engineering, \\ Linköping University, SE-581 83 Linköping, SWEDEN
}

\begin{abstract}
A compressor model is developed. It is capable of representing mass flow and pressure characteristic for three different regions: surge, normal operation as well as for when the compressor acts as a restriction, i.e. having a pressure ratio of less than unity. Different submodels are discussed and methods to parametrize the given model structure are given. Both the parametrization and validation are supported extensively by measured data. Dynamic data sets include measurements from engine and surge test stands. The compressor model is further validated against a database of stationary compressor maps. The proposed model is shown to have good agreement with measured data for all regions, without the need for extensive geometric information or data.
\end{abstract}

Keywords: automobile powertrains, engine control, parameter estimation, restriction, turbo

\section{INTRODUCTION AND MOTIVATION}

A compressor model capable of handling all operating modes is beneficial when investigating control strategies and controller design for turbocharged (TC) engines. The operating modes to cover are: normal operation, compressor surge and choke, or restriction, operation.

Surge is a dangerous instability and can occur e.g. during a gear shift under acceleration, where a throttle closing causes a fast reduction in mass flow. The mass flow reduction produces an increase in pressure ratio over the compressor and if the turbo does not slow down fast enough the pressure ratio will be too high and the mass flow will revert through the compressor. This lowers the pressure, and the compressor is then again able to build pressure. This increases the pressure ratio and the surge cycle can then be repeated. The oscillating mass flow can damage, and even destroy, the turbo and can cause undesired noise.

Choke can lead to a pressure drop over the compressor. A single stage turbo compressor can be forced to operate with a pressure drop over it during short transients, before it has overcome inertia effects. For a two stage system this can also be obtained if the bypass valve does not function properly, or is forced closed when charging with the first compressor stage. A passively controlled bypass valve, that opens when a pressure drop is created over it, will always give a pressure ratio of less than unity for the second stage, when the high pressure stage is bypassed.

\subsection{Contribution}

Compressor models describing and validated over all three regions simultaneously are, to the authors knowledge, not found in the literature. This paper describes the development and validation of a novel compressor model and its integration in an existing Mean Value Engine Model (MVEM), see Hendricks (1989). The developed and implemented model is parametrized and validated using experimental data.

\subsection{Mean Value Engine Modeling}

Component based MVEM of TC spark ignited (SI) engines is outlined in Eriksson et al. (2002) and Eriksson (2007). The baseline model is developed and validated in Andersson (2005) and has been extended to handle surge in Bergström and Leufven (2007). The extension utilizes the model by Moore-Greitzer (Greitzer, 1981) that introduces a compressor mass flow state. A comparison of compressor modeling techniques can be found in Moraal and Kolmanovsky (1999).

The TCSI model structure, see Figure 1, uses restrictions (air filter, compressor, intercooler, throttle, engine, turbine, exhaust system) interconnected with control volumes, and further extended with surge and wastegate valves. There are 14 states: six pairs of control volume pressures and temperatures, turbo shaft speed and compressor mass flow.

\subsection{Experimental Data}

Experimental data is collected from three different platforms: a compressor driven by a separate electric motor in a surge test stand (Andersen et al., 2008), a compressor as part of a single stage turbocharger installed in a test stand, and a two stage turbocharged engine mounted in an engine test stand.

Compressor maps SAE-corrected maps (SAE, 1995a,b), and externally measured maps, are used in the evaluation. Most maps use corrected mass flow and shaft speed

$$
\begin{aligned}
& \bar{N}=\frac{N_{t c}}{\sqrt{\mathrm{T} / \mathrm{T}_{\text {std }}}} \\
& \bar{W}=W \frac{\sqrt{\mathrm{T} / \mathrm{T}_{\text {std }}}}{\mathrm{p} / \mathrm{p}_{\text {std }}}
\end{aligned}
$$

where $p_{\text {std }}$ and $T_{\text {std }}$ are referred to as reference conditions, and are provided together with the map data. The bar indicates a corrected quantity, and the compressor model of this paper is given in corrected quantities. The compressor 


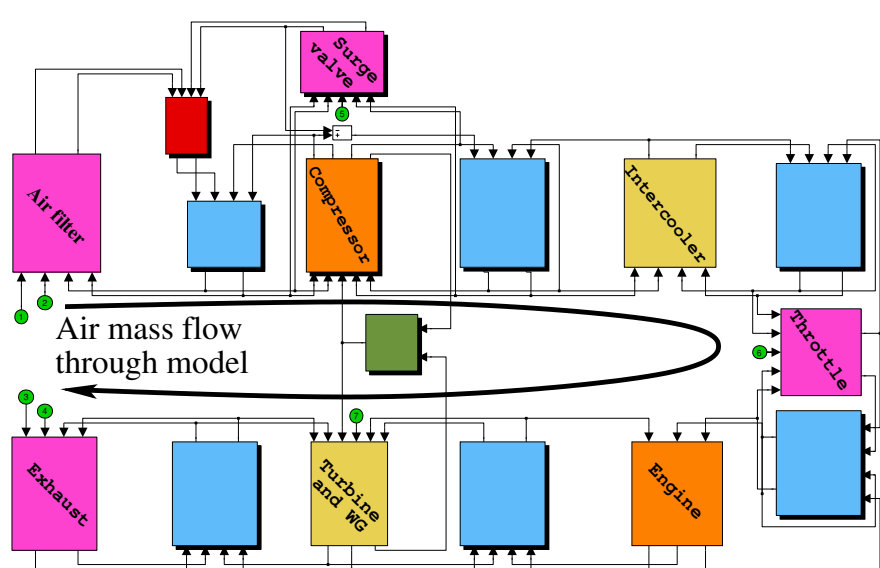

Fig. 1. Surge capable MVEM. The mass flow path starts in the air filter and is indicated by the arrow. The different components are interconnected with control volumes. The turbocharger shaft is seen between the compressor and the turbine.

map, see e.g. Figure 5, shows what stationary pressure ratio, $\Pi_{c}=\mathrm{p}_{c} / \mathrm{p}_{\mathrm{af}}$, and adiabatic efficiency, $\eta_{c}$, a compressor achieves as a function of $\bar{W}$ and $\bar{N}$. Here $p_{c}$ and $p_{a f}$ are the pressures in the control volumes before and after the compressor. Points of equal $\bar{N}$ are normally connected, giving a compressor speed line, SpL. Points are measured from the smallest mass flow, found at the surge line, $\mathrm{SuL}$, to the largest mass flow where choking can occur.

\subsection{Surge properties}

This paper considers full surge, where the compressor mass flow, $W_{c}<0$ during parts of a surge cycle. To characterize a surge cycle, the following features are proposed

$t_{s c}[\mathrm{~s}] \quad$ Cycle time of a surge cycle

$\Gamma_{\Pi_{c s}}[-] \quad\left(\Pi_{\mathrm{c}, \max }-\Pi_{\mathrm{c}, \min }\right) /\left(\Pi_{\mathrm{c}, \max }-1\right)$

where $\Pi_{c, \max }$ and $\Pi_{c, \min }$ are the largest and smallest $\Pi_{c}$ during a surge cycle. These are exemplified using engine test stand measurements in Figure 2, that also shows pressure and shaft speed variations for a typical surge cycle.

\section{COMPRESSOR MODEL}

To include surge capability in the model, the approach in Greitzer (1981) is followed, introducing a state for the compressor mass flow $W_{c}$ according to

$$
\frac{d W_{c}}{d t}=\frac{\pi D_{c}^{2}}{4 L_{c}} \cdot\left(\hat{p}-p_{c}\right), \quad \hat{p}=\hat{\Pi} \cdot p_{a f}
$$

where $D_{c}$ is the compressor diameter, $L_{c}$ is the duct length. $p_{c}$ and $p_{a f}$ are set by the system, while $\hat{p}$ (and $\hat{\Pi}$ ) is a measure of the pressure the compressor builds given an operating point, and only for stationary operation is $p_{c}=\hat{p}$.

The compressor model has also sub models describing: outlet temperature, $T_{c}$, and consumed torque, $T q_{c}$

$$
\begin{aligned}
T_{c} & =T_{a f}\left(\frac{\Pi_{c}^{\frac{\gamma-1}{\gamma}}-1}{\eta_{c}}+1\right) \\
T q_{c} & =30 / \pi \frac{\left(T_{c}-T_{a f}\right) c_{p} W_{c}}{N_{t c}}
\end{aligned}
$$

where $\gamma, c_{p}$ are gas properties taken as constants due to the small variations in inlet gas properties, and $\eta_{c}$ is the

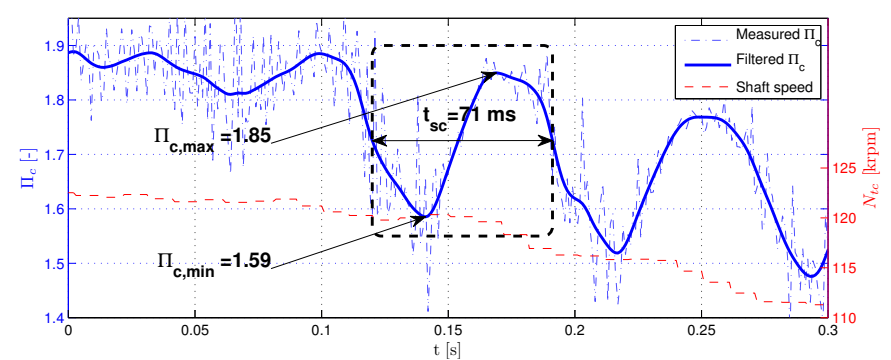

Fig. 2. Engine dynamometer measurements showing $\Pi_{c}$ for two surge cycles. Solid line is zero phase shift low pass filtered $\Pi_{c}$ to easier separate the measurement signal (dash dotted) from the noise. Dashed line shows turbo shaft speed in $[\mathrm{krpm}]$. The dashed box shows one surge cycle. The surge properties are: $t_{s c}=71 \mathrm{~ms}$ and $\Gamma_{\Pi_{c s}}=(1.85-1.59) /(1.85-1)=31 \%$.

compressor adiabatic efficiency. The dynamics for the shaft speed $N_{t c}$ is given by the turbo shaft torque balance

$$
\frac{d\left(N_{t c} \pi / 30\right)}{d t}=\frac{1}{J_{t c}}\left(T q_{t}-T q_{c}-T q_{t c, \text { fric }}\right)
$$

where $T q_{t}$ is torque supplied from the turbine, $T q_{t c, \text { fric }}$ is the shaft friction and $J_{t c}$ is the turbocharger inertia.

\section{ELLIPSE COMPRESSOR Пิ-MODEL}

To model $\hat{\Pi}$, a subdivision is made dependent on if $\bar{W}$ is larger or smaller than the SuL mass flow $\bar{W}_{\mathrm{SuL}}$, and if $\bar{W}<0$. SuL for the model is defined as the point on a $\mathrm{SpL}$ with positive mass flow that has zero slope.

To motivate the model consider the following. A normal SpL found in maps has close to zero slope at SuL, and then falls of with increasing slope for increasing $\bar{W}$. The shape of a SpL is similar to an ellipse. This is used as the basis of the proposed Ellipse model, where the major and minor axis as well as the center point are allowed to vary with $\bar{N}$. With increasing use of two stage turbo systems, models capable of representing also the flow restriction characteristic $\left(\Pi_{c}<1\right)$ of the compressor are needed, see Figure 3. Single stage turbo systems can also operate with $\Pi_{c}<1$, e.g. during a transient before the turbo has spun up and built up pressure. An Ellipse model SpL includes this region. $\hat{\Pi}$ for $\bar{W}<0$ is modeled with a turbine like behavior. The model equations for the different regions are

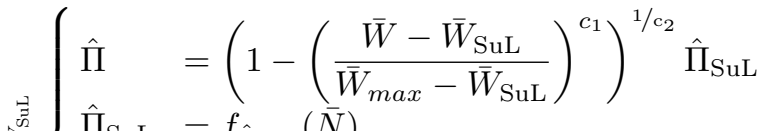

$$
\begin{aligned}
& \hat{\Pi}_{\mathrm{SuL}}=f_{\hat{\Pi}_{\mathrm{SuL}}}(\bar{N}) \\
& \wedge \quad \bar{W}_{\mathrm{SuL}}=f_{\bar{W}_{\mathrm{SuL}}}(\bar{N}) \\
& =\bar{W}_{\text {max }}=f_{\bar{W}_{\text {max }}}(\bar{N}) \\
& c_{j} \quad=f_{c_{j}}(\bar{N}), j=1,2 \\
& \begin{array}{l}
\hat{\Pi}=\hat{\Pi}_{c 0}+b_{2} \bar{W}^{2}+b_{3} \bar{W}^{3} \\
\hat{\Pi}_{c 0}=\hat{\Pi}_{\mathrm{SuL}}-f_{\Gamma_{\Pi_{c s}}}(\bar{N})\left(\hat{\Pi}_{\mathrm{SuL}}-1\right)
\end{array} \\
& b_{2}=3\left(\hat{\Pi}_{\mathrm{SuL}}-\hat{\Pi}_{\mathrm{c} 0}\right) / \overline{\mathrm{W}}_{\mathrm{SuL}}^{2} \\
& \stackrel{b^{2}}{b_{3}}=-2\left(\hat{\Pi}_{\mathrm{SuL}}-\hat{\Pi}_{\mathrm{co}}\right) / \overline{\mathrm{W}}_{\mathrm{SuL}}^{3}
\end{aligned}
$$

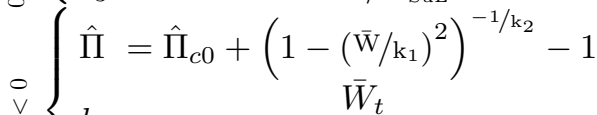

$$
\begin{aligned}
& \stackrel{\vee}{\vee}\left\{k_{1}=\frac{W_{t}}{\sqrt{1-\left(\hat{\Pi}_{c t}-\hat{\Pi}_{c 0}+1\right)^{-k_{2}}}}\right.
\end{aligned}
$$




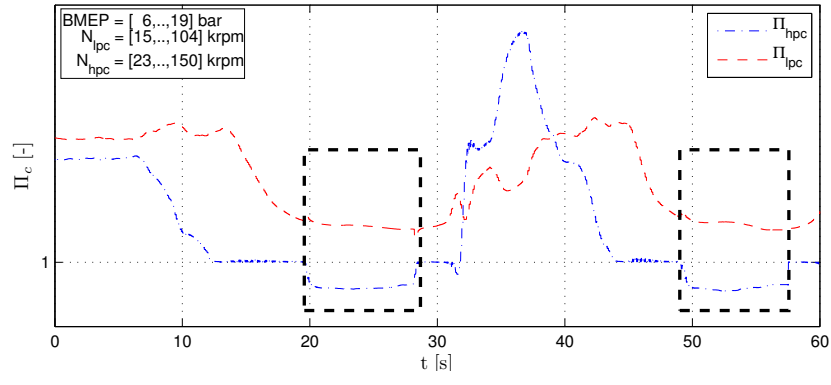

Fig. 3. Measured $\Pi_{c}$ for both the first and the second stage of a two stage system. Regions having $\Pi_{h p c}<1$ are marked with boxes. The data motivates the need for a compressor model that also handles $\Pi_{c}<1$.

Note that some of the model parameters have a physical meaning: maximum corrected compressor mass flow at zero pressure ratio $\left(\bar{W}_{\max }\right)$, at the surge line $\left(\bar{W}_{\mathrm{SuL}}\right)$, and pressure ratio at the surge line $\left(\hat{\Pi}_{\mathrm{SuL}}\right)$. The function $f_{\Gamma_{\Pi_{c s}}}$ describes one of the surge properties proposed in Section 1.4, and determines the pressure build up at zero mass flow, $\hat{\Pi}_{c 0}$. The parameters $c_{1}, c_{2}$ define the ellipse eccentricity. The semi-diameters of the ellipse are given by the map points $\left(\bar{W}_{\mathrm{SuL}}, \hat{\Pi}_{\mathrm{SuL}}\right),\left(\bar{W}_{\mathrm{SuL}}, 0\right)$ and $\left(\bar{W}_{\max }, 0\right)$.

The following subsections will describe and motivate the functions $f_{i}$, were

$$
i \in\left\{c_{1}, c_{2}, \bar{W}_{\max }, \bar{W}_{\mathrm{SuL}}, \hat{\Pi}_{\mathrm{SuL}}, \Gamma_{\Pi_{c s}}\right\}
$$

\subsection{Ellipse $\hat{\Pi}$ parametrization $-\bar{W}>\bar{W}_{\text {SuL }}$}

Both $\bar{W}_{\text {SuL }}$ and $\hat{\Pi}_{\text {SuL }}$ are expected to increase with $\bar{N}$. For $\bar{N}=0, \bar{W}_{\text {SuL }}=0$ and $\hat{\Pi}_{\text {SuL }}=1$ are expected. An increasing $\bar{W}_{\max }$ with increasing $\bar{N}$ is expected, until the inducer chokes, see Gravdahl and Egeland (1997).

Functions $f_{c_{1}}$ and $f_{c_{2}}$ The first two Ellipse model functions enable an $\bar{N}$-dependent eccentricity of the ellipse (compare $\left.\left(\left(\overline{\mathrm{W}}-\overline{\mathrm{W}}_{\mathrm{SuL}}\right) /\left(\overline{\mathrm{W}}_{\max }-\overline{\mathrm{W}}_{\mathrm{SuL}}\right)\right)^{c_{1}}+\left(\hat{\Pi} / \hat{\Pi}_{\mathrm{SuL}}\right)^{c_{2}}=1\right)$. The following $f_{c_{1}}$ and $f_{c_{2}}$ were found to balance model simplicity and descriptive capabilities for the map database

$$
\begin{aligned}
& f_{c_{1}}(\bar{N})=c_{1,0}+c_{1,1} \bar{N} \\
& f_{c_{2}}(\bar{N})=c_{2,0}+c_{2,1} \bar{N}^{c_{2,2}}
\end{aligned}
$$

where $c_{i, j}$ are real valued constants.

Function $f_{\bar{W}_{\max }} \quad$ A first order polynomial is proposed to model maximum corrected mass flow $\bar{W}_{\max }$

$$
f_{\bar{W}_{\text {max }}}(\bar{N})=c_{3,0}+c_{3,1} \bar{N}
$$

where $c_{3,0}>0$ gives a positive flow also for $\bar{N}=0$. A minimum selector can be added to physically correspond to a choked inducer flow, $\bar{W}_{c i}$, according to

$$
\tilde{f}_{\bar{W}_{\max }}=\min \left(\bar{W}_{c i}, f_{\bar{W}_{\max }}\right)
$$

This was not needed for the any of compressors studied.

Functions $f_{\bar{W}_{S u L}}$ and $f_{\hat{\Pi}_{S u L}} \quad$ For surge mass flow

$$
f_{\bar{W}_{\mathrm{SuL}}}(\bar{N})=0+c_{4,1} \bar{N}^{c_{4,2}}
$$

and for surge pressure ratio

$$
f_{\hat{\Pi}_{\mathrm{SuL}}}(\bar{N})=1+c_{5,1} \bar{N}^{c_{5,2}}
$$

are proposed, where the 0 and 1 emphasize zero flow and unity pressure ratio for $\bar{N}=0$.
The parameters in (7)-(10) are determined using a nonlinear least square method on a compressor map.

\subsection{Ellipse $\hat{\Pi}$ parametrization $-\bar{W}<\bar{W}_{\text {SuL }}$}

First it is recognized that the exact SpL shape, for $\bar{W} \in$ $\left[0, \bar{W}_{\mathrm{SuL}}\right]$, is not of great importance for the surge behavior, see Hansen et al. (1981). It can also be seen in (3), where the change in $p_{c}$ is given by the emptying and filling dynamic of the downstream control volume, and $\hat{p}$ is given by the compressor model. The slope of a $\mathrm{SpL}$ is positive in this region, Galindo et al. (2008); Greitzer (1981), and the difference $\left(\hat{p}-p_{c}\right)$ is therefore large, and the region is traversed fast. The, for surge, important parameter $\hat{\Pi}_{c 0}$ has been recognized in e.g. Theotokatos and Kyrtatos (2001), but is here instead modeled as a function of the parameter $\Gamma_{\Pi_{c s}}$, see (6).

Importance of $\hat{\Pi}_{c 0} \quad$ The importance of $\hat{\Pi}_{c 0}$ comes from the fact that the $\Pi_{c}$-trajectory, during deep surge, has to enclose the point $\left(\hat{\Pi}_{c 0}, \bar{W}=0\right)$. This is seen in the second row of (3), i.e.

$$
\frac{d W_{c}}{d t}=\frac{\pi D_{c}^{2}}{4 L_{c}} \cdot\left(\hat{p}-p_{c}\right)
$$

where $\hat{p}$ has to lead $p_{c}$ to increase $W_{c}$ and take the compressor out of reversed flow. The trajectory is given by the compressor characteristics and the system properties.

To find $\hat{\Pi}_{c 0}$ consider the following. To get $\frac{d W_{c}}{d t}=1 \mathrm{~kg} / \mathrm{s}^{2}$ a difference of $\left(\hat{p}-p_{c}\right) \approx 0.8 \mathrm{kPa}$ is needed, given reasonable values of $D_{c}=0.04 \mathrm{~m}, L_{c}=1 \mathrm{~m}$. Since $0.8<<200 \mathrm{kPa}$, the $\Pi_{c}$-trajectory will pass closely under the $\left(\hat{\Pi}_{c 0}, 0\right)$-point, when the compressor is moving out of reversed mass flow. This close connection $\hat{\Pi}_{c 0} \approx \Pi_{c 0}$ means that measurement data of $\Pi_{c}$ during surge can be used to assess $\Gamma_{\Pi_{c s}}$. The approximation $\hat{\Pi}_{c 0} \approx \Pi_{c 0}$ needs to be checked for a given application though, since making the $\left(\pi \mathrm{D}_{\mathrm{c}}^{2}\right) / 4 \mathrm{~L}_{\mathrm{c}}$-factor small means that the difference $\left(\hat{p}-p_{c}\right)$ has to be increased, for a given value of $\frac{W_{c}}{d t}$. Further, the pressure $\hat{p}_{0}=p_{a f} \hat{\Pi}_{c 0}$ is the pressure of the downstream control volume needed for the compressor to go from negative to positive $W_{c}$, which will affect $t_{s c}$.

Effect from $\Gamma_{\Pi_{c s}}, \bar{W}_{t}, \hat{\Pi}_{c t}, k_{2}$ on the surge properties $k_{1}$ is given by algebraic constraints (6) forcing all SpL through $\left(\bar{W}_{t}, \hat{\Pi}_{c t}\right)$. To investigate how the other Ellipse model parameters affect the surge properties from Section 1.4, a model of the surge test rig is used. Each of the parameters $\Gamma_{\Pi_{c s}}, \bar{W}_{t}, \hat{\Pi}_{c t}, k_{2}$ are varied $\pm\{10 \%, 30 \%\}$ from nominal values of $\Gamma_{\Pi_{c s}}=49 \%, \bar{W}_{t}=-0.059, \hat{\Pi}_{c t}=10$ and $k_{2}=0.5$. The resulting sensitivity in the surge properties are summarized in Table 1 and measured surge is compared to a simulation with the nominal values in Figure 7 . The most important parameters are found to be $\Gamma_{\Pi_{c s}}$ and $\bar{W}_{t}$.

Surge rig data $\Gamma_{\Pi_{c s}}$ is calculated from continuous surge measurements. The test rig is further described in Andersen et al. (2008), and some of the data is presented in Table 2. $\Gamma_{\Pi_{c s}}$ is found to be $\approx 50 \%$ for a wide range of $\bar{N}$ for this compressor. Further, if several data sets with $\bar{N}=$ const are studied in more detail, it is found that once deep surge is established, $\Gamma_{\Pi_{c s}}$ shows only a small dependence of mean 


\begin{tabular}{c|c|c|c|c|c} 
& $\Gamma_{\Pi_{c s}}$ & $\bar{W}_{t}$ & $\hat{\Pi}_{c t}$ & $k_{2}$ & $\begin{array}{c}\text { Param. } \\
\text { change }\end{array}$ \\
\hline \multirow{2}{*}{$\Delta t_{s c}$} & $19 \%(-20 \%)$ & $-4 \%(5 \%)$ & $0 \%(-1 \%)$ & $-1 \%(1 \%)$ & $+30 \%(-30 \%)$ \\
& $6 \%(-7 \%)$ & $-1 \%(1 \%)$ & $0 \%(-0 \%)$ & $-0 \%(0 \%)$ & $+10 \%(-10 \%)$ \\
\hline$\Delta \Gamma_{\Pi_{c s}}$ & $26 \%(-27 \%)$ & $1 \%(-1 \%)$ & $-0 \%(0 \%)$ & $0 \%(-0 \%)$ & $+30 \%(-30 \%)$ \\
& $9 \%(-9 \%)$ & $0 \%(-0 \%)$ & $-0 \%(0 \%)$ & $0 \%(-0 \%)$ & $+10 \%(-10 \%)$ \\
\hline
\end{tabular}

Table 1. Sensitivity of the surge properties with respect to changes in the Ellipse model parameters. The parameters are varied $\pm\{10 \%, 30 \%\}$ from nominal, $t_{s c}=81 \mathrm{~ms}$ and $\Gamma_{\Pi_{c s}}=54 \%$.

\begin{tabular}{cc|cc|c}
$N_{t c}[\mathrm{krpm}]$ & mean $\left(W_{t h}\right)[\mathrm{g} / \mathrm{s}]$ & $\Pi_{c, \max }[-]$ & $\Pi_{c, \text { min }}[-]$ & $\Gamma_{\Pi_{c s}}[-]$ \\
\hline 80 & 15.0 & 1.35 & 1.17 & $51 \%$ \\
91 & 17.8 & 1.45 & 1.23 & $49 \%$ \\
99 & 21.9 & 1.53 & 1.26 & $51 \%$ \\
110 & 25.0 & 1.66 & 1.33 & $50 \%$ \\
120 & 28.5 & 1.79 & 1.39 & $50 \%$ \\
131 & 30.4 & 1.96 & 1.48 & $50 \%$ \\
140 & 38.5 & 2.10 & 1.54 & $51 \%$ \\
\hline 130 & 49.0 & 1.89 & 1.88 & $2 \%$ \\
130 & 42.9 & 1.90 & 1.87 & $3 \%$ \\
131 & 37.9 & 1.94 & 1.82 & $13 \%$ \\
131 & 33.0 & 1.94 & 1.48 & $49 \%$ \\
131 & 30.4 & 1.96 & 1.48 & $50 \%$ \\
\hline
\end{tabular}

Table 2. Surge test stand measurements in normal and continuous surge operation. Upper part: full surge at different $\bar{N} \cdot \Gamma_{\Pi_{c s}} \approx 50 \%$ independent of $\bar{N}$. Lower part: one $\bar{N}$ with different mean $\left(W_{t h}\right)$. The two last rows show that, for full surge, an increase in mean $\left(W_{t h}\right)$ with $10 \%$ only decreases $\Gamma_{\Pi_{c s}}$ with $<1 \%$.
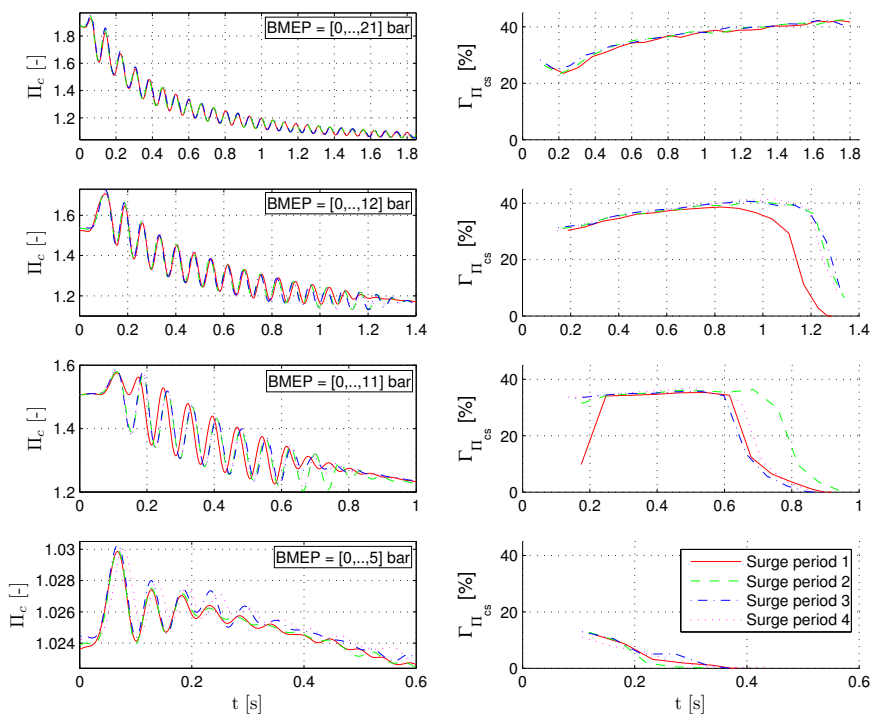

Fig. 4. Measured surge in an engine test stand. Each row shows multiple surge periods initiated from the same engine operating point. Initial $\Pi_{c}$ decreases from top to bottom. Left: $\Pi_{c}$-traces. Right: Calculated $\Gamma_{\Pi_{c s}}$. The surge repeatability is remarkable.

mass flow. The third last row of Table 2 shows $\Gamma_{\Pi_{c s}}$ for mild surge.

Engine test stand data Figure 4 presents typical surge measurements at tip-out from an engine test stand. It can be seen that when deep surge is established, surge is repeatable with remarkable accurary, although the behavior of the first and last cycles of the surge periods can differ, where some cycles show a mild surge behavior. The right plots show $\Gamma_{\Pi_{c s}}$, where a increasing trend in $\Gamma_{\Pi_{c s}}$ for decreasing $\Pi_{c}$ can be seen. When the surge period is over $\Gamma_{\Pi_{c s}}$ goes to zero. Since $N_{t c}$ decreases during the tip-outs, it is not possible to get a correct value of $\Gamma_{\Pi_{c s}}$. $\Pi_{c, \max }$ of Section 1.4 is here taken as the peak following an associated $\Pi_{c, \text { min }}$-value, giving an approximation of $\Gamma_{\Pi_{c s}}$. The motivation for choosing the peak afterward is that $N_{t c}$ is approximately constant during the time from a $\Pi_{c}$-dip to the following $\Pi_{c}$-peak, see Figure 8 . An increasing trend in $\Gamma_{\Pi_{c s}}$ can be seen for decreasing $\Pi_{c}$.

No surge data The experimental data indicates that $\Gamma_{\Pi_{c s}} \approx 35 \%$ is a good approximation if no surge data exists. For compressor maps having points with lower mass flow than (9), another method can be used to shape the speed lines. Given the model structure from (6), nonlinear optimization can be used to find the parameters of $f_{\hat{\Pi}_{c 0}}$ to obtain best fit to the map points.

\section{4. $\eta_{C}$-MODEL}

The six parameter efficiency model, $\eta_{c, 6 p}$, presented in Andersson (2005) is used in this paper

$$
\left\{\begin{aligned}
\eta_{c, 6 p} & =\eta_{c_{\max }}-\chi^{T} Q \chi \\
\chi & =\left[\begin{array}{c}
\bar{W}-\bar{W}_{\eta_{c_{\max }}} \\
\sqrt{\Pi_{c}-1}-\left(\hat{\Pi}_{\eta_{c_{\max }}}-1\right)
\end{array}\right] \\
Q & =\left[\begin{array}{ll}
Q_{11} & Q_{12} \\
Q_{21} & Q_{22}
\end{array}\right], Q_{12}=Q_{21}
\end{aligned}\right.
$$

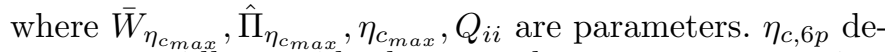
creases rapidly outside the normal compressor operating region. Other $\eta_{c}$ models can be found in e.g. Jensen et al. (1991).

For the restriction operating region $\left(\Pi_{c}<1\right)$, and for the surge region a constant efficiency, $\eta_{c, r / s}$, is assumed to maintain simplicity of the model. The rapid decrease of the $\eta_{c, 6 p}$-model outside the normal operating region of the compressor is used to further maintain simplicity of the full $\eta_{c}$-model. For the $\eta_{c}$-model, restrictive or surging $(\mathrm{r} / \mathrm{s})$ operation is assumed when $\eta_{c, 6 \text { param }}<\eta_{c, r / s}$. The efficiency model can therefore be made up by taking the maximum value of $\eta_{c, r / s}$ and $\eta_{c, 6 p}$. A continuous $\eta_{c^{-}}$ function is also obtained. The complete efficiency model is given by

$$
\eta_{c}=\max \left(\eta_{c, 6 p}, \eta_{c, r / s}\right)
$$

\section{$4.1 \eta_{c}$ parametrization}

Normal and restrictive region $\quad \bar{W}_{\eta_{\max }}, \hat{\Pi}_{\eta_{\max }}, \eta_{c_{\max }}$ and $Q_{i i}$ of (11) are estimated using nonlinear optimization on compressor map data.

Looking at available data for the region $\Pi_{c}<1$, a small temperature increase can in fact be seen. This increase is however rather small, $2-4 \mathrm{~K}$. Since the gas expands through the compressor a temperature decrease is expected but this can be counteracted by heat transfer effects. A constant efficiency of $20 \%$ is used here, which is also supported in Müller et al. (2005).

Surge region Hints for the compressor efficiency in the surge region can be found in compressor inlet temperature measurements, and by studying the shaft speed variations during surge. However, a good estimate is hard to achieve due to the slow temperature sensors. This paper proposes to model the efficiency as a constant, since this is found to produce temperature signals with good agreement with 

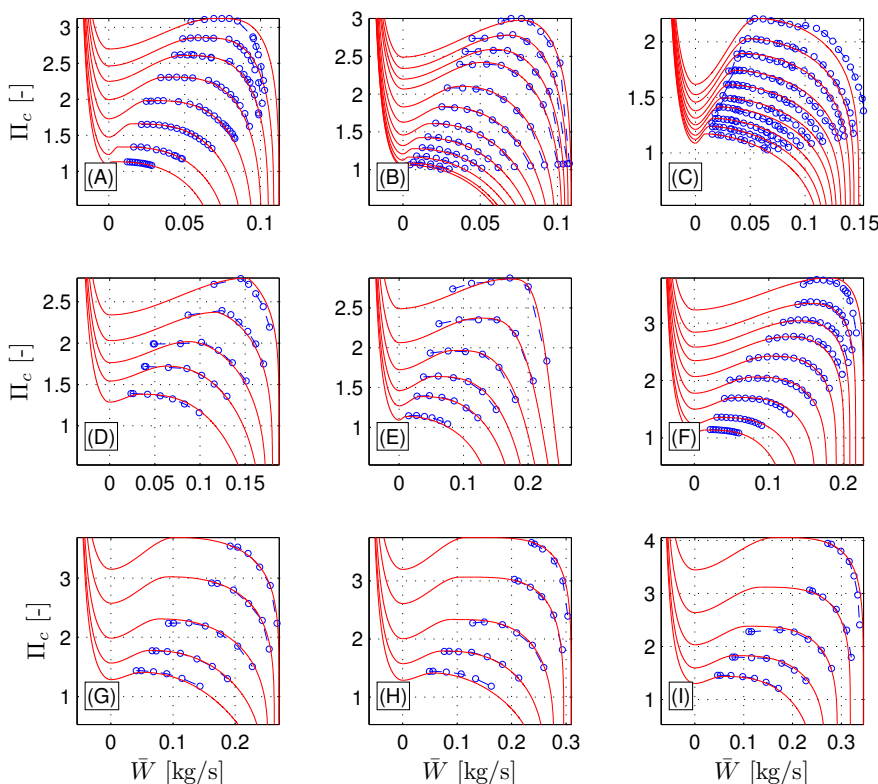

Fig. 5. Validation of Ellipse model on nine (A-I) compressor maps. Circles represent map data. The maps range from small $(\approx 100 \mathrm{~g} / \mathrm{s})$ compressors to large $(\approx 350 \mathrm{~g} / \mathrm{s})$ and from both single as well as two stage setups. The overall performance of the model is good over the entire normal operating region.

\begin{tabular}{r|rrr|rrr} 
& \multicolumn{3}{|c|}{$\eta_{c}>60 \%$} & \multicolumn{3}{c}{$\eta_{c}<60 \%$} \\
\hline Map & $\operatorname{mean}(\mathrm{e})$ & std(e) & $\max (|e|)$ & $\operatorname{mean}(\mathrm{e})$ & $\operatorname{std}(\mathrm{e})$ & $\max (|e|)$ \\
\hline (A) & $0.32 \%$ & $2.66 \%$ & $14.22 \%$ & $1.04 \%$ & $5.98 \%$ & $23.06 \%$ \\
(B) & $-0.03 \%$ & $1.11 \%$ & $2.78 \%$ & $0.55 \%$ & $5.89 \%$ & $31.75 \%$ \\
(C) & $0.00 \%$ & $3.51 \%$ & $21.55 \%$ & $0.77 \%$ & $10.74 \%$ & $72.34 \%$ \\
(D) & $0.37 \%$ & $2.43 \%$ & $8.04 \%$ & $-2.31 \%$ & $1.91 \%$ & $4.24 \%$ \\
(E) & $0.09 \%$ & $0.72 \%$ & $1.60 \%$ & $-1.33 \%$ & $2.42 \%$ & $4.42 \%$ \\
(F) & $0.22 \%$ & $2.25 \%$ & $10.14 \%$ & $-0.80 \%$ & $6.48 \%$ & $22.17 \%$ \\
(G) & $0.02 \%$ & $1.39 \%$ & $3.05 \%$ & $2.11 \%$ & $4.22 \%$ & $6.13 \%$ \\
(H) & $-0.25 \%$ & $2.01 \%$ & $6.06 \%$ & $-1.26 \%$ & $9.70 \%$ & $13.29 \%$ \\
(I) & $0.53 \%$ & $2.85 \%$ & $8.40 \%$ & $5.62 \%$ & $29.14 \%$ & $61.80 \%$
\end{tabular}

Table 3. Mean, standard deviation and magnitude of relative error between modeled pressure ratio and compressor map data, $e=$ $\left(\hat{\Pi}-\Pi_{\text {map }}\right) / \hat{\Pi}$, for nine representative maps. Map points with $\eta_{c}>60 \%$ (left) and $\eta_{c}<60 \%$ (right). The model performance is good, with increasing relative error for decreasing $\eta_{c}$.

measurements. A constant efficiency of $20 \%$ is assumed here, which is also supported in Grigoriadis (2008).

\section{VALIDATION}

The model validation is presented first for П⿵and $\eta_{c}$, followed by a full system validation. Due to data availability the same data is used for both parametrization and validation. More maps were used in the evaluations than presented here, providing further support for the model.

\subsection{Ellipse $\hat{\Pi}$ validation}

$\hat{\Pi}$ - Normal region The Ellipse model performance is validated for nine representative maps in Figure 5, and further summarized in Table 3 . The automated scripts produce a model with an average relative error of less than $1 \%$, for most points. The largest errors are found for choked mass flows, where the error can be up to $20 \%$.

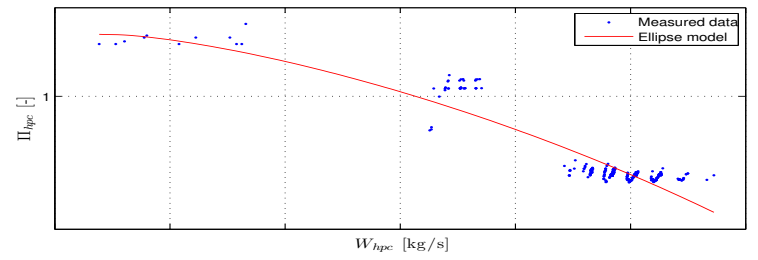

Fig. 6. Measurements and simulation of $\Pi_{c}<1$ operation. The model representation for $\Pi_{c}<1$ is good.

\begin{tabular}{r|rrr|rrr} 
& \multicolumn{3}{|c|}{$\eta_{c}>60 \%$} & \multicolumn{3}{c}{$\eta_{c}<60 \%$} \\
\hline Map & $\operatorname{mean}(\mathrm{e})$ & $\mathrm{std}(\mathrm{e})$ & $\max (|e|)$ & $\operatorname{mean}(\mathrm{e})$ & $\mathrm{std}(\mathrm{e})$ & $\max (|e|)$ \\
\hline (A) & $-0.29 \%$ & $1.82 \%$ & $4.76 \%$ & $0.68 \%$ & $4.01 \%$ & $8.11 \%$ \\
(B) & $0.04 \%$ & $4.48 \%$ & $10.85 \%$ & $20.38 \%$ & $30.82 \%$ & $76.66 \%$ \\
(C) & $0.10 \%$ & $2.36 \%$ & $8.39 \%$ & $0.06 \%$ & $7.64 \%$ & $24.40 \%$ \\
(D) & $-0.50 \%$ & $3.06 \%$ & $8.44 \%$ & $1.24 \%$ & $4.33 \%$ & $8.25 \%$ \\
(E) & $0.00 \%$ & $3.83 \%$ & $8.65 \%$ & $0.18 \%$ & $10.09 \%$ & $24.16 \%$ \\
(F) & $-0.37 \%$ & $2.16 \%$ & $6.77 \%$ & $1.21 \%$ & $3.57 \%$ & $8.78 \%$ \\
(G) & $-0.29 \%$ & $1.86 \%$ & $5.56 \%$ & $1.50 \%$ & $6.83 \%$ & $9.90 \%$ \\
(H) & $-0.35 \%$ & $3.55 \%$ & $12.63 \%$ & $1.77 \%$ & $6.42 \%$ & $10.35 \%$ \\
(I) & $-0.33 \%$ & $2.41 \%$ & $6.70 \%$ & $1.76 \%$ & $7.50 \%$ & $10.40 \%$ \\
\hline
\end{tabular}

Table 4. Mean, standard deviation and magnitude of relative error $\left(e=\left(\eta_{\mathrm{c}}-\eta_{\text {map }}\right) / \eta_{\mathrm{c}}\right)$ between modeled $\eta_{c}$, and compressor map data for nine compressor maps. Left: Points with $\eta_{c}>60 \%$. Right: Points with $\eta_{c}<60 \%$. The model performance is good. Data set (B) contains $\mathrm{SuL}$ measured down to the gas stand discharge flow characteristics (see Figure 5), with small values of $\eta_{c}$, causing large relative errors due to the model's lower limit $\eta_{c, r / s}$.

$\hat{\Pi}<1-$ Restriction quadrant There does not exist much data from compressors operated in this region. The Ellipse model performance is shown in Figure 6, where the agreement with measured data is shown to be good.

\subsection{Efficiency model validation}

Efficiency - Normal region The same compressor maps as in Figure 5, are used to validate the $\eta_{c}$ model, and the result is presented in Table 4. Good performance is obtained for the normal region with $\eta_{c}>60 \%$, with increasing discrepancy for points with lower efficiency.

\subsection{Integration performance validation}

The developed and parametrized compressor model is incorporated into the MVEM structure developed in Eriksson (2007). The MVEM library components are used to build both a surge test and a full TCSI engine model.

Surge test stand The surge test stand model consists of a compressor, two control volumes and a restriction. These are parametrized to correspond to the geometries and physical behavior from measurements. The results are shown in Fig. 7, where it is seen that measured and modeled $t_{s c}$ and pressure oscillations are in good agreement.

Full engine MVEM surge The full TCSI MVEM developed in Andersson (2005) is extended with the Ellipse compressor model. The same engine used to parametrize the MVEM, was driven in surge and measured. These measurements are here compared to the extended MVEM. The measured and modeled compressor control volume pressure are in good agreement, see Figure 8. 

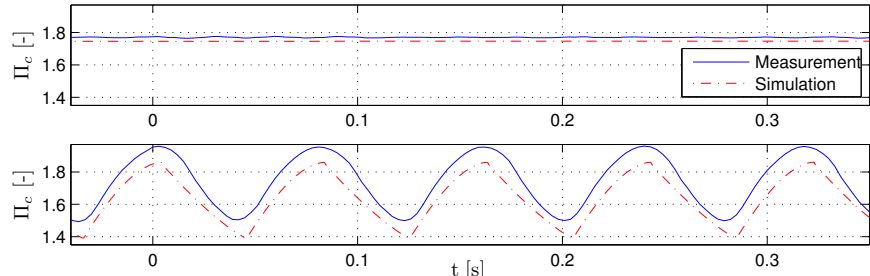

Fig. 7. Modeled and measured surge rig $\Pi_{c}$, for a constant $\bar{N}=130 \mathrm{krpm}$ for two $W_{t h}$. Upper: $W_{t h}$ close to surge. Lower: Operating point with full surge. The modeled $t_{s c}$ and $\Gamma_{\Pi_{c s}}$ are in good agreement.

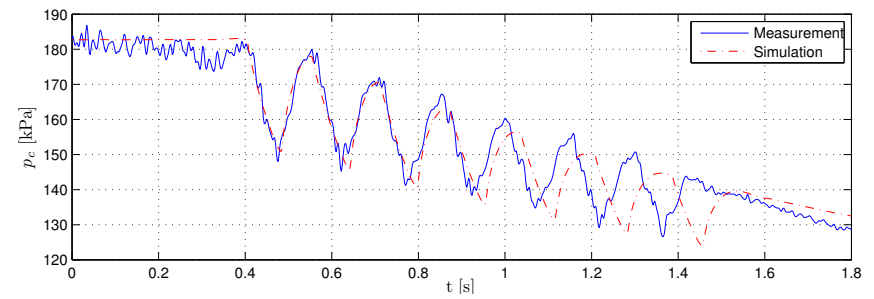

Fig. 8. Engine test stand measured and MVEM simulated compressor control volume pressure for a surge transient. The pressure oscillations are well modeled both in amplitude and frequency.

\section{CONCLUSIONS}

Normal and choke compressor operation can be modeled using ellipses, and extended to describe surge. The parametrization can be automated given a compressor map and data from surge. The proposed basis functions for the curve fitting are validated on a database of compressor maps, and are shown to give good description for a wide range of automotive compressors, both single stage and two stage. The basis function are physically motivated, and ensures a sound extrapolation capability. The ellipse model has good performance for normal operation, as well as for surge and operation with pressure ratio of less than unity.

The conclusions of the surge rig and engine test stand experiments are: If no available surge data exists, a constant value of $\Gamma_{\Pi_{c s}} \in[30,50] \%$ will suffice. Further, deep surge cycles are highly repeatable. $\Gamma_{\Pi_{c s}}$ shows only a small dependency of mean mass flow. Surge pressure oscillations can be seen in measured data down to pressure ratios of close to unity, and are not limited to high pressure ratios/high shaft speeds. Turbo shaft speed is almost constant when going from negative to positive compressor mass flow. The deep surge cycle frequency is mainly given by the emptying and filling dynamics in combination with the compressor characteristics, where pressure build up at zero mass flow is most important.

\section{REFERENCES}

J. Andersen, F. Lindström, and F. Westin. Surge Definitions for Radial Compressors in Automotive Turbochargers. SAE Int. J. of Engines, 1(1):218, 2008.

P. Andersson. Air Charge Estimation in Turbocharged Spark Ignition Engines. $\mathrm{PhD}$ thesis 989, Linköping University, 2005.

J. Bergström and O. Leufven. Surge modeling and control of automotive turbochargers. Master's thesis LiTH-ISYEX-3999, Linköping University, 2007.
L. Eriksson. Modeling and control of turbocharged SI and DI engines. Oil \& Gas Science and Technology - Rev. IFP, 62(4):523-538, 2007.

L. Eriksson, L. Nielsen, J. Brugård, J. Bergström, F. Pettersson, and P. Andersson. Modeling of a turbocharged SI engine. Annual Reviews in Control, 26(1):129 - 137, 2002. ISSN 1367-5788.

J. Galindo, J.R. Serrano, H. Climent, and A. Tiseira. Experiments and modelling of surge in small centrifugal compressor for automotive engines. Exp. Thermal and Fluid Science, 32(3):818 - 826, 2008. ISSN 0894-1777.

J.T. Gravdahl and O. Egeland. Speed and surge control for a low order centrifugal compressor model. IEEE Int. Conf. on Control Applications, pages 344 -349, 1997.

E. M. Greitzer. The stability of pumping systems - The 1980 Freeman Scholar Lecture. ASME Trans. J. of Fluids Engineering, 103:193-242, 1981.

P. Grigoriadis. Experimentelle Erfassung und Simulation instantionärer Verdichterphänomene bei Turboladern von Fahrzeugmotoren. $\mathrm{PhD}$ thesis, Technischen Universität Berlin, 2008.

K. E. Hansen, P. Jorgensen, and P. S. Larsen. Experimental and theoretical study of surge in a small centrifugal compressor. ASME J. Fluids Engineering, (103):391395, 1981.

E. Hendricks. The analysis of mean value engine models. SAE 890563, 1989.

J.-P. Jensen, A.F. Kristensen, S.C. Sorenson, N. Houbak, and E. Hendricks. Mean value modeling of a small turbocharged diesel engine. SAE 910070, 1991.

P. Moraal and I. Kolmanovsky. Turbocharger modeling for automotive control applications. SAE 1999-01-0908, 1999.

M. Müller, S. Sumser, P. Fledersbacher, K. Rößler, K. Fieweger, and H.J. Bauer. Using the centrifugal compressor as a cold-air turbine. 8th Int. conf. on turbochargers and turbocharging, London, 2005.

SAE. SAE J1826 - Turbocharger Gas Stand Test Code. SAE standard, 1995a.

SAE. SAE J922 - Turbocharger Nomenclature and Terminology. SAE standard, 1995b.

G. Theotokatos and N.P. Kyrtatos. Diesel engine transient operation with turbocharger compressor surging. SAE 2001-01-1241, 2001.

\section{Appendix A. NOMENCLATURE}

\begin{tabular}{ll}
\hline Symbol & Description \\
\hline$a f$ & Air filter \\
$c$ & Compressor \\
$c 0$ & Compressor at zero flow \\
$c s$ & Compressor surge \\
$c t$ & Compressor as turbine \\
$c, 6 p$ & 6 parameter model \\
$f r i c$ & Friction \\
$h p c$ & High pressure stage \\
$l p c$ & Low pressure stage \\
max & Maximum \\
$\Pi_{c, \text { max } / \text { min }}$ & Max/Min $\Pi_{c}$ during sc \\
$r / s$ & Restriction or surge \\
$\mathrm{SpL}$ & Speed line \\
$\mathrm{SuL}$ & Surge line \\
$s c$ & Surge cycle \\
$s t d$ & Standard \\
$t$ & Turbine \\
$t c$ & Turbocharger \\
$t h$ & Throttle \\
\hline
\end{tabular}

\begin{tabular}{ll}
\hline Symbol & Description \\
\hline$\eta$ & Adiabatic efficiency \\
$p$ & Pressure \\
$\Pi$ & Pressure ratio \\
$\hat{p}$ & Pressure build up \\
$\hat{\Pi}=\hat{\mathrm{p}} / \mathrm{paf}_{\text {af }}$ & Pressure build up ratio \\
$\Gamma$ & $\Pi$-ratio during surge \\
$T$ & Temperature \\
$T q$ & Torque \\
$W$ & Mass flow \\
$\bar{W}$ & Corrected mass flow \\
$N$ & Shaft speed \\
$\bar{N}$ & Corrected shaft speed \\
$D_{c}, L_{c}$ & Charact. dia. \& length \\
$e$ & Relative error \\
$\gamma, c_{p}$ & Gas properties \\
$J$ & Inertia \\
$t$ & Time \\
\hline
\end{tabular}

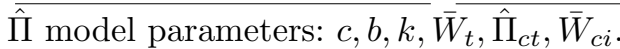

$\eta_{c}$ model parameters: $Q, \eta_{c, r / s}, \eta_{c, \max }$. 